\title{
Slow light in photonic crystals with loss or gain
}

\author{
Andrey A. Sukhorukov and Thomas P. White \\ Nonlinear Physics Centre and Centre for Ultrahigh-bandwidth Devices for Optical Systems \\ (CUDOS), Australian National University, Canberra, ACT 0200, Australia
}

\begin{abstract}
We develop a perturbation theory for slow-light photonic-crystal waveguides engineered to suppress groupvelocity dispersion, and predict that weak material loss (gain) is enhanced proportionally to the slow-down factor, whereas the attenuation (amplification) rate saturates for loss (gain) exceeding a certain threshold. This happens due to hybridization of propagating and evanescent modes which allows significant intensity enhancement observed in our numerical simulations for photonic crystal waveguides even under strong material losses.
\end{abstract}

Keywords: Slow light, photonic crystal, material loss

\section{INTRODUCTION}

Enhancement of light-matter interactions in slow light (SL) waveguides provides opportunities for tunable delays, switching, and monitoring of optical pulses in compact photonic structures. ${ }^{1}$ SL occurs naturally for optical frequencies at the edges of photonic transmission bands, however in this regime group-velocity dispersion (GVD) is practically unavoidable leading to undesirable pulse broadening. ${ }^{2}$ The GVD can be suppressed in disperion engineered waveguides which support SL inside an optical transmission band, as was successfully demonstrated for photonic-crystal waveguides ${ }^{3}$ and suggested for plasmonic guiding structures. ${ }^{4}$ Importantly, optical pulses can be coupled very efficiently into dispersion-engineered slow-light waveguides, ${ }^{5}$ even in the "frozen light" regime at zero group velocity. ${ }^{6}$

Slow light also enhances the effects of loss or gain, and it is important to understand the limitations of device performance due to pulse attenuation or the potential for achieving pulse amplification. In photonic crystals, scattering losses can arise due to fabrication imperfections. ${ }^{7}$ On the other hand, loss can appear due to material properties, such as absorption in metals for plasmonic waveguides or gases in hollow fibers. Alternatively, loss or gain can be introduced through resonant interactions with a pump beam, for example through Raman processes. ${ }^{1}$ For band-edge SL, the presence of loss modifies the dispersion and limits the maximum achievable slow-down factor, ${ }^{8-10}$ which in turn limits the absorption enhancement. It was shown that scattering losses in dispersion engineered SL waveguides can be significantly lower than for equivalent band-edge SL, ${ }^{7}$ which raises a question about a possible difference in the effect of material loss.

In this work, we reveal through analytical analysis and numerical simulations the generic effects of loss or gain on wave propagation in dispersion-engineered SL waveguides, uncovering unique features distinct from band edge SL considered previously. ${ }^{8-10}$ In particular, we predict loss saturation due to hybridization of propagating and evanescent waves, which can provide pulse intensity enhancement even under strong losses. In Sec. 2, we review the properties of engineered photonic-crystal waveguides which support slow-light modes with zero groupvelocity dispersion. In Sec. 3 we develop a perturbation theory and describe analytically the effects of material loss or gain in such waveguides. Then, in Sec. 4 we present the results of finite-difference time-domain simulations that confirm analytical predictions, and additionally demonstrate the intensity enhancement effect. We present conclusions and outlook in Sec. 5. 
(a)
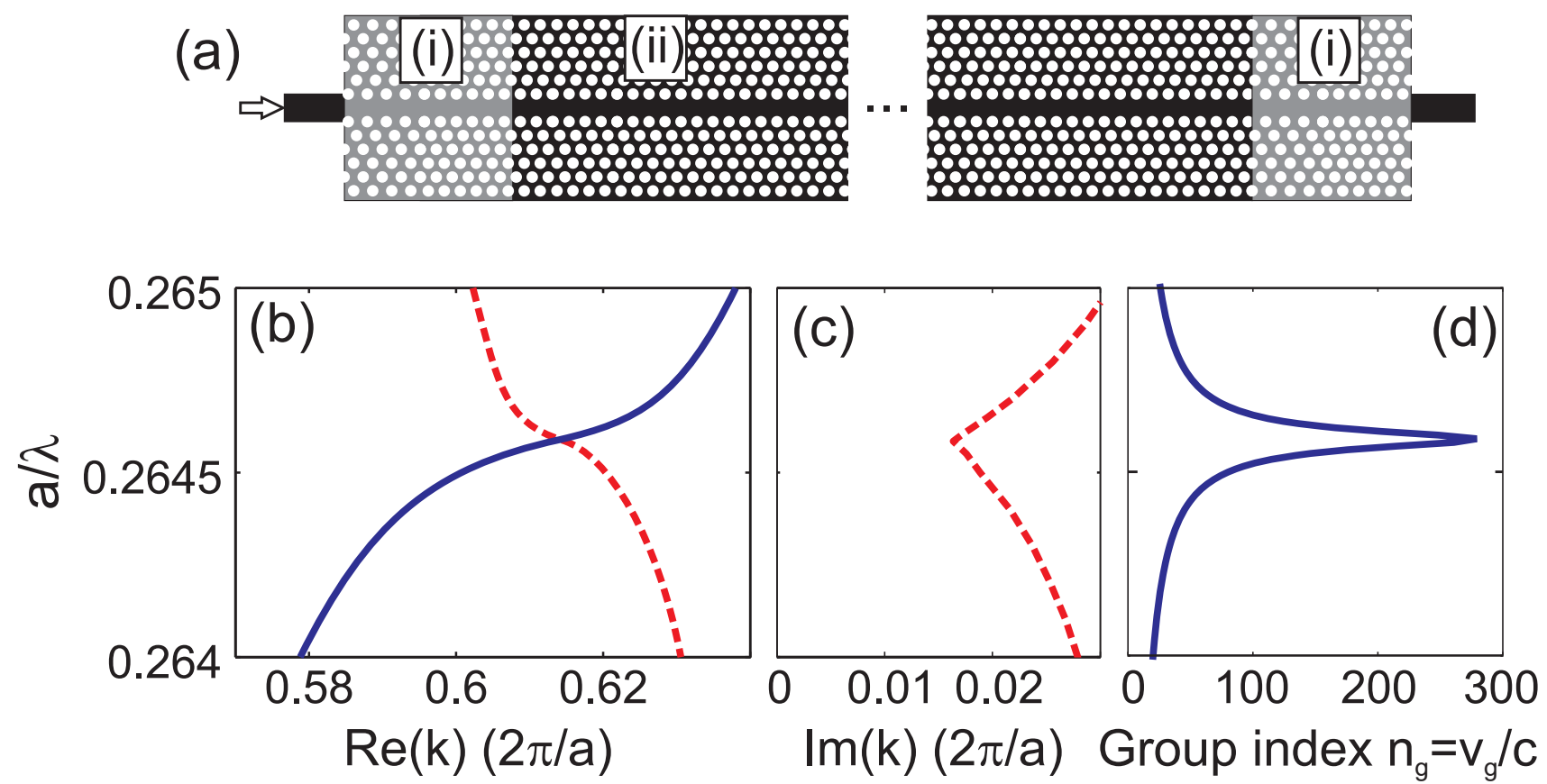

Figure 1. (Color online) (a) $\mathrm{PhC}$ waveguide geometry. (b) $\mathrm{Re}(\mathrm{k})$ dispersion curves for the propagating mode (solid) and first evanescent mode (dashed) in the slow light waveguide. (c) Corresponding $\operatorname{Im}(\mathrm{k})$ curve for the evanescent mode. (c) Group index of the propagating mode, where the slow light region is associated with the dispersion inflection point.

\section{DISPERSION-ENGINEERED SLOW-LIGHT WAVEGUIDES}

As a starting point, we consider a SL waveguide in which the dispersion $\omega(k)$ is engineered to suppress GVD, i.e. $\alpha=\partial^{2} \omega / \partial k^{2}=0$ at the dispersion point $\omega_{0}=\omega\left(k_{0}\right)$, where $\omega$ is frequency and $k$ is wavenumber. Then, a Taylor expansion of the dispersion curve to third order can be written as

$$
\omega(k) \simeq \omega_{0}+v_{g, 0}\left(k-k_{0}\right)+\beta\left(k-k_{0}\right)^{3},
$$

where $v_{g, 0}=\partial \omega /\left.\partial k\right|_{k_{0}}$ is the group velocity, and $\beta=\left.(1 / 6)\left(\partial^{3} \omega / \partial k^{3}\right)\right|_{k_{0}}$. The absence of the second-order term is due to GVD suppression, associated with an inflection point in the dispersion relation. We demonstrate such dispersion in a specially designed photonic crystal $(\mathrm{PhC})$ waveguide shown in Fig. 1(a). The slow light section, (ii), consists of a two-dimensional $\mathrm{PhC}$ slab with refractive index $n=2.83$, period $a$ and holes of radius $r=0.3 a$. A missing line of holes forms the waveguide core, and the first two rows of holes either side are shifted parallel to the waveguide axis by $0.3 a$ and $0.425 a$ respectively. The fundamental TE-polarized mode dispersion has an inflection point, see solid curve in Fig. 1(b), where the group index (slow-down factor) is $n_{g} \approx 280$ in a narrow wavelength range, see Fig. $1(\mathrm{~d})$. The dispersion is well approximated by Eq. (1) with $k_{0} a /(2 \pi)=0.6131$, $\omega_{0} a /(2 \pi c)=0.264585, v_{g, 0}=c / 280$, and $\beta=16 c(2 \pi / a)^{2}$.

\section{ANALYTICAL PERTURBATION THEORY}

To study the influence of material loss (or gain) on the waveguide dispersion, we consider the effect of a small imaginary part of the refractive index, $n=n_{r}+i n_{i}$. The corresponding complex permittivity is given by $\epsilon \simeq \epsilon_{r}+i \epsilon_{i}$, where $\epsilon_{r}$ is that of the lossless waveguide, and $\epsilon_{i}=2 n_{r} n_{i}$. From first-order perturbation theory, ${ }^{9}$ the small change in $\epsilon$ produces a small frequency shift

$$
\Delta \omega=-i \frac{1}{2} \omega f \frac{\epsilon_{i}}{\epsilon_{r}}
$$

Here

$$
f=\frac{\left\langle E\left|\epsilon_{i}\right| E\right\rangle_{V}}{\left\langle E\left|\epsilon_{r}\right| E\right\rangle}
$$

Further author information available at the Nonlinear Physics Centre web site: http://physics.anu.edu.au/nonlinear 


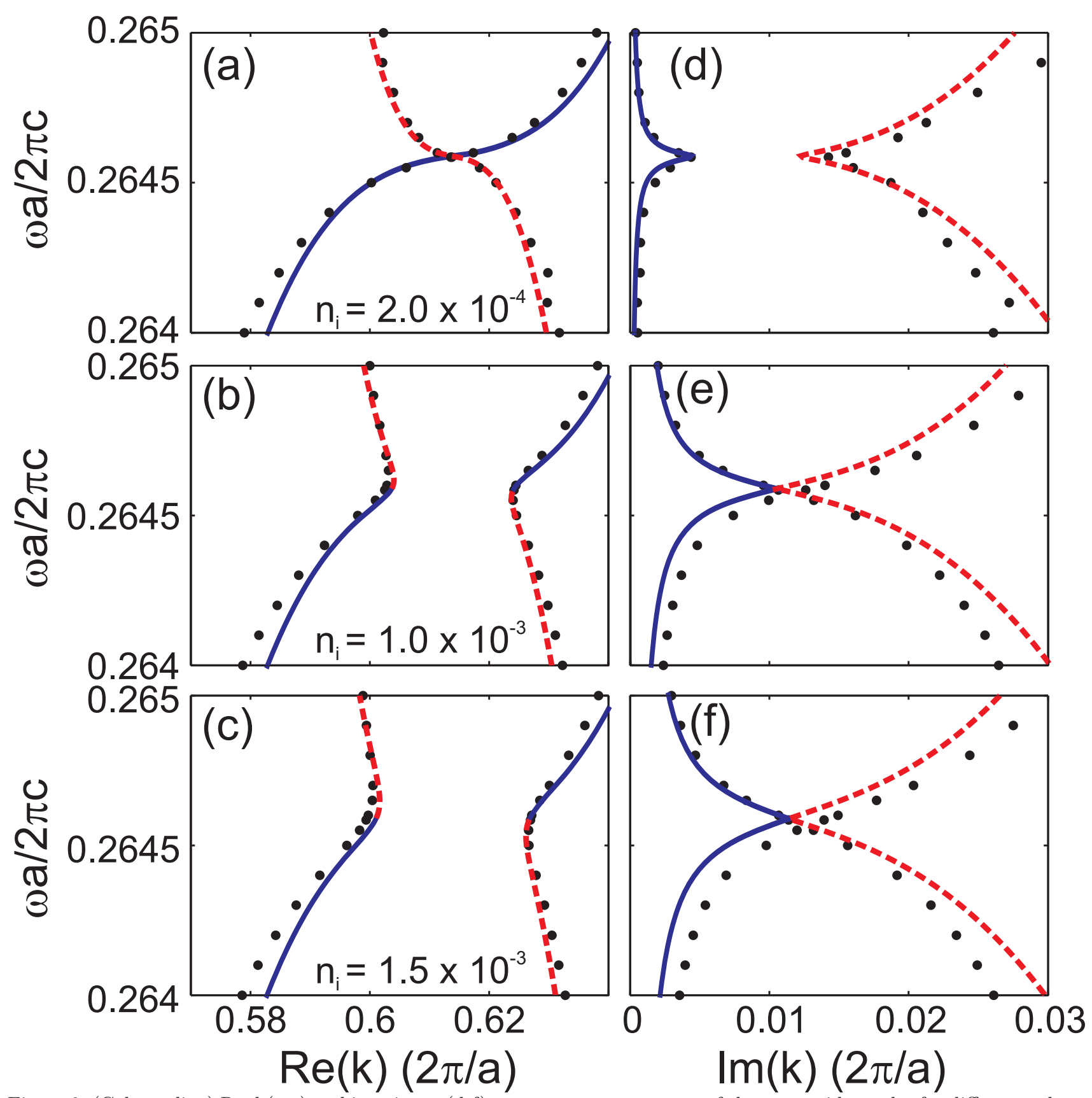

Figure 2. (Color online) Real (a-c) and imaginary (d-f) wavevector components of the waveguide modes for different values of loss. The solid and dashed curves are calculated using the perturbation model and dots are numerical FDTD results. The line type follows the mode classification in the absence of losses as either propagating (solid) or evanescent (dashed).

describes the proportion of the total mode energy in the volume $V$ comprising the lossy dielectric, where $E$ is the electric field mode profile. Then, we replace $\omega_{0}$ in Eq. (1) with $\omega_{0}+\Delta \omega_{0}$, where $\Delta \omega_{0}$ is given by Eq. (2) evaluated at $\omega_{0}$, and invert the resulting equation to solve for $k(\omega)$ at real $\omega$. The function $\omega(k)$ is cubic, and hence there are three solutions at each frequency. When there is no loss $\left(n_{i}=0\right)$, we obtain one real $k_{1}$ and a pair of complex solutions, $k_{2}=k_{3}^{*}$, which correspond to evanescent modes. The dispersion of the evanescent mode which decays in the propagation direction (with $\operatorname{Im}(\mathrm{k}) \geq 0$ ) is plotted as dashed curves in Figs. 1(a) and (b).

We now analyze the effect of loss on the waveguide dispersion. All three solutions $k(\omega)$ are complex for $n_{i}>0$, but only two have $\operatorname{Im}(\mathrm{k})>0$ corresponding to a physically relevant situation of wave decay in the propagation 

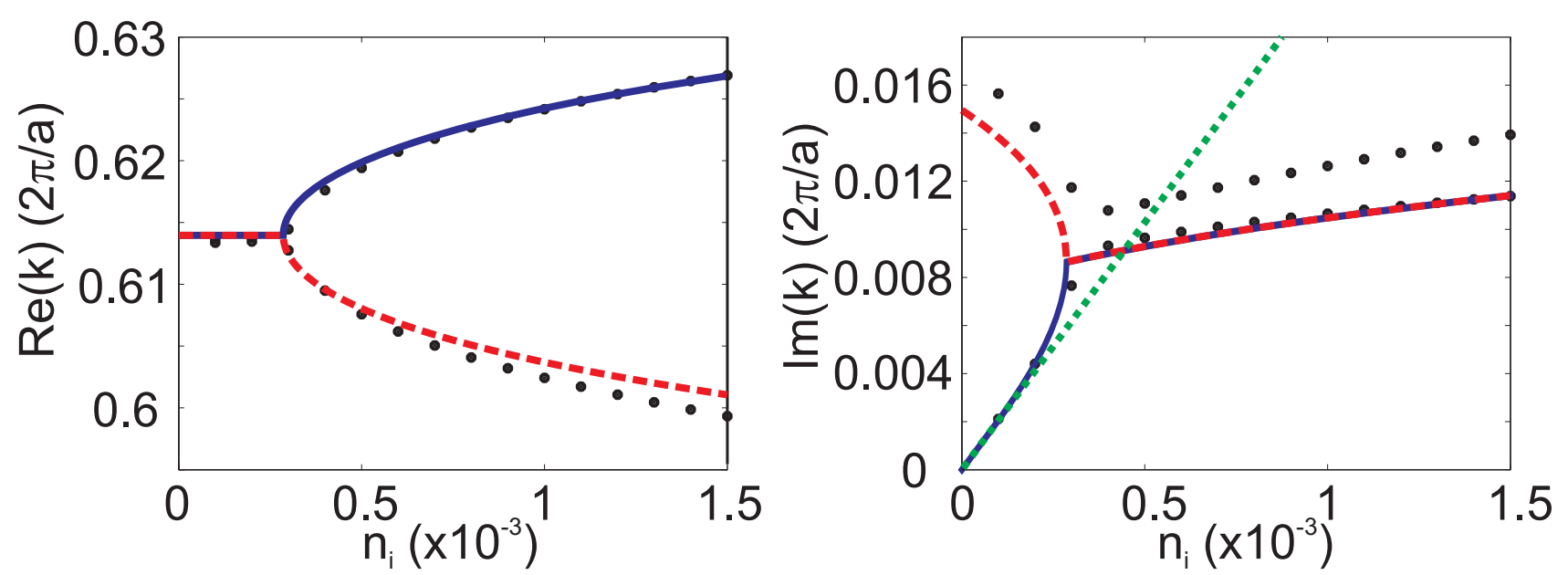

Figure 3. (Color online) Real (a) and imaginary (b) wavevector components of the waveguide modes at the inflection point frequency plotted as a function of $n_{i}$.

direction. We show dispersion curves for the two modes for a set of increasing $n_{i}$ in Fig. 2 . When $n_{i}=2 \times 10^{-4}$, dispersion of $\operatorname{Re}(\mathrm{k})$ in Fig. 2(a) closely resembles the lossless case in Fig. 1(b), apart from a slight distortion close to the inflection point. On the other hand, what was previously the propagating mode with purely real $k$ now has a small $\operatorname{Im}(\mathrm{k})$ component that peaks at the inflection point frequency due to slow light enhancement of the loss, see Fig. 2(d). As $n_{i}$ increases, the propagating and evanescent modes form hybrid states close to the inflection point as shown in Figs. 2(b,e) and (c,f). This coupling manifests as an avoided crossing of the Re(k) dispersion curves and a corresponding crossing of the $\operatorname{Im}(\mathrm{k})$ curves, such that the decay rate of both modes is equal at $\omega_{0}$. Note also that the inflection feature of the original dispersion curve in Fig. 1(b) has disappeared, dramatically changing the dispersion and slow light properties of the waveguide in the vicinity of $\omega_{0}$. From the analytic form of the cubic solutions of $k(\omega)$, we find that the loss threshold for the mode hybridization is

$$
\epsilon_{i}^{\mathrm{th}}>\left(\frac{3}{\beta}\right)^{1 / 2} \frac{4 \epsilon_{r} v_{g, 0}^{3 / 2}}{9 \omega_{0}},
$$

which corresponds to $\epsilon_{i}^{\text {th }}=1.2 \times 10^{-3}$ or $n_{i}^{\text {th }}=2.2 \times 10^{-4}$ for the SL PhC waveguide shown in Fig. 1(a).

The mode hybridization is evident in the dependencies of mode dispersion at the inflection point frequency $\left(\omega_{0}\right)$ shown in Fig. 3 . When the loss is below the threshold value, both modes have equal $\operatorname{Re}(\mathrm{k})$, as for the lossless case, while the values of $\operatorname{Im}(\mathrm{k})$ approach each other as $\epsilon_{i} \rightarrow \epsilon_{i}^{\text {th }}$. Once the loss passes the threshold value, the $\operatorname{Re}(\mathrm{k})$ curves split, while $\operatorname{Im}(\mathrm{k})$ is equal for the two modes. For small losses below the threshold, perturbation theory predicts enhancement of the propagating mode decay rate proportional to the slow-down factor,

$$
\operatorname{Im}(\mathrm{k}) \simeq \frac{1}{2} \omega_{0} \mathrm{f} \frac{\epsilon_{\mathrm{i}}}{\epsilon_{\mathrm{r}} \mathrm{v}_{\mathrm{g}, 0}},
$$

and we show this dependence by the dotted green line in Fig. 3(b). Above the threshold, the decay rate saturates, remaining below the level of

$$
\operatorname{Im}(\mathrm{k}) \simeq \sqrt{\frac{\mathrm{v}_{\mathrm{g}, 0}}{3 \beta}}+\frac{1}{12} \omega_{0} \mathrm{f} \frac{\epsilon_{\mathrm{i}}-\epsilon_{\mathrm{i}}^{\mathrm{th}}}{\epsilon_{\mathrm{r}} \mathrm{v}_{\mathrm{g}, 0}} .
$$

Note that here the multiplier in front of $\epsilon_{i}$ is decreased six times compared to the case of small losses. Since our analytical results do not depend on the sign of $\epsilon_{i}$, we can similarly predict the effect of amplification saturation in the case of material gain.

\section{FINITE-DIFFERENCE TIME-DOMAIN SIMULATIONS}

To verify the accuracy of the simple perturbation model, we performed two-dimensional FDTD simulations of propagation in $\mathrm{PhC}$ waveguides with weak material absorption. The geometry for these simulations is shown in 
Fig. 1(a). Light is launched from a narrow ridge waveguide into $\mathrm{PhC}$ waveguide (i) before entering the slow light waveguide (ii). Section (i) supports a relatively fast mode with $n_{g} \approx 5$ at $\omega_{0}$, and provides efficient coupling to the ridge waveguide and to section (ii). ${ }^{5,11}$ Here, waveguide (i) is formed by increasing the lattice constant of waveguide (ii) by $7 \%$ in the direction parallel to the waveguide axis. Light is coupled out of the waveguide in an identical way after propagation through 100 periods of waveguide (ii).

To calculate the dispersion properties of waveguide (ii) we launch a broad frequency spectrum pulse and perform a temporal Fourier transform to extract the steady-state fields for frequencies near the inflection point. We then apply a Bloch-mode extraction technique to simultaneously extract both the complex wavevector components and field profiles of all the waveguide modes. ${ }^{12}$

The results of the FDTD dispersion extraction are plotted as dots in Figs. 2 and 3, where lines correspond to perturbation model results. Recall that the perturbation model takes as input only the unperturbed dispersion of the propagating mode and the modal overlap with the lossy material $(f)$, Eqs. (2) and (3). We determine that $f=0.77$ using the mode profile at the inflection point frequency. The agreement with the numerical simulation is remarkable; the simple model reproduces all key features of the mode hybridization, both qualitatively and quantitatively. The only minor deviation is seen in Fig. 3(b) where the imaginary wavevector components extracted from the FDTD simulations are not exactly equal in the region $\epsilon_{i}>\epsilon_{i}^{\text {th }}$ whereas they are equal in the analytical model.

The numerical simulations allow us to investigate whether lossy slow light waveguides of this type could still provide the increased intensities which can enhance nonlinear effects. This is a nontrivial question, since not only does the loss directly reduce the field intensity inside the waveguide, but the reshaping of the dispersion curves changes the group velocity. Figure 4 shows the maximum intensity enhancement at the inflection point as a function of $n_{i}$. The enhancement is defined as the ratio of the maximum value of $\left|H_{y}\right|^{2}$ in the slow light region to that in the input waveguide, where $H_{y}$ is the magnetic field component perpendicular to the plane of the $\mathrm{PhC}$. The figures on the right show the field profiles along the waveguide for several values of $n_{i}$. The results show that it is possible to achieve an order of magnitude enhancement in the waveguide even in the presence of loss on the scale of $n_{i}=10^{-3}$. While such high loss would clearly not allow long propagation distances, it may still be possible to observe enhanced nonlinear effects such as third-harmonic generation, which has been measured in the first few microns of silicon slow light $\mathrm{PhC}$ waveguides. ${ }^{13}$

\section{CONCLUSIONS}

In conclusion, we have analyzed the influence of loss or gain on slow-light associated with an inflection point of mode dispersion. We have developed a simple perturbation model which predicts the hybridization of propagating and evanescent waves and associated saturation of loss or gain. This effect is confirmed in numerical simulations, which also enables strong field enhancement even in the presence of strong losses. These results have a general nature, and we anticipate their application in studies and design of slow-light waveguides based on photoniccrystal slabs, fibers, and plasmonic structures.

This work was supported by the Australian Research Council through Centre of Excellence CUDOS and Discovery projects.

\section{REFERENCES}

1. J. B. Khurgin and R. S. Tucker, eds., Slow Light: Science and Applications, Tailor and Francis, New York, 2009.

2. R. J. P. Engelen, Y. Sugimoto, Y. Watanabe, J. P. Korterik, N. Ikeda, N. F. van Hulst, K. Asakawa, and L. Kuipers, "The effect of higher-order dispersion on slow light propagation in photonic crystal waveguides," Opt. Express 14, pp. 1658-1672, Feb 202006.

3. M. D. Settle, R. J. P. Engelen, M. Salib, A. Michaeli, L. Kuipers, and T. F. Krauss, "Flatband slow light in photonic crystals featuring spatial pulse compression and terahertz bandwidth," Opt. Express 15, pp. 219-226, Jan 82007.

4. A. Karalis, J. D. Joannopoulos, and M. Soljacic, "Plasmonic-dielectric systems for high-order dispersionless slow or stopped subwavelength light," Phys. Rev. Lett. 103, pp. 043906-4, Jul 242009. 

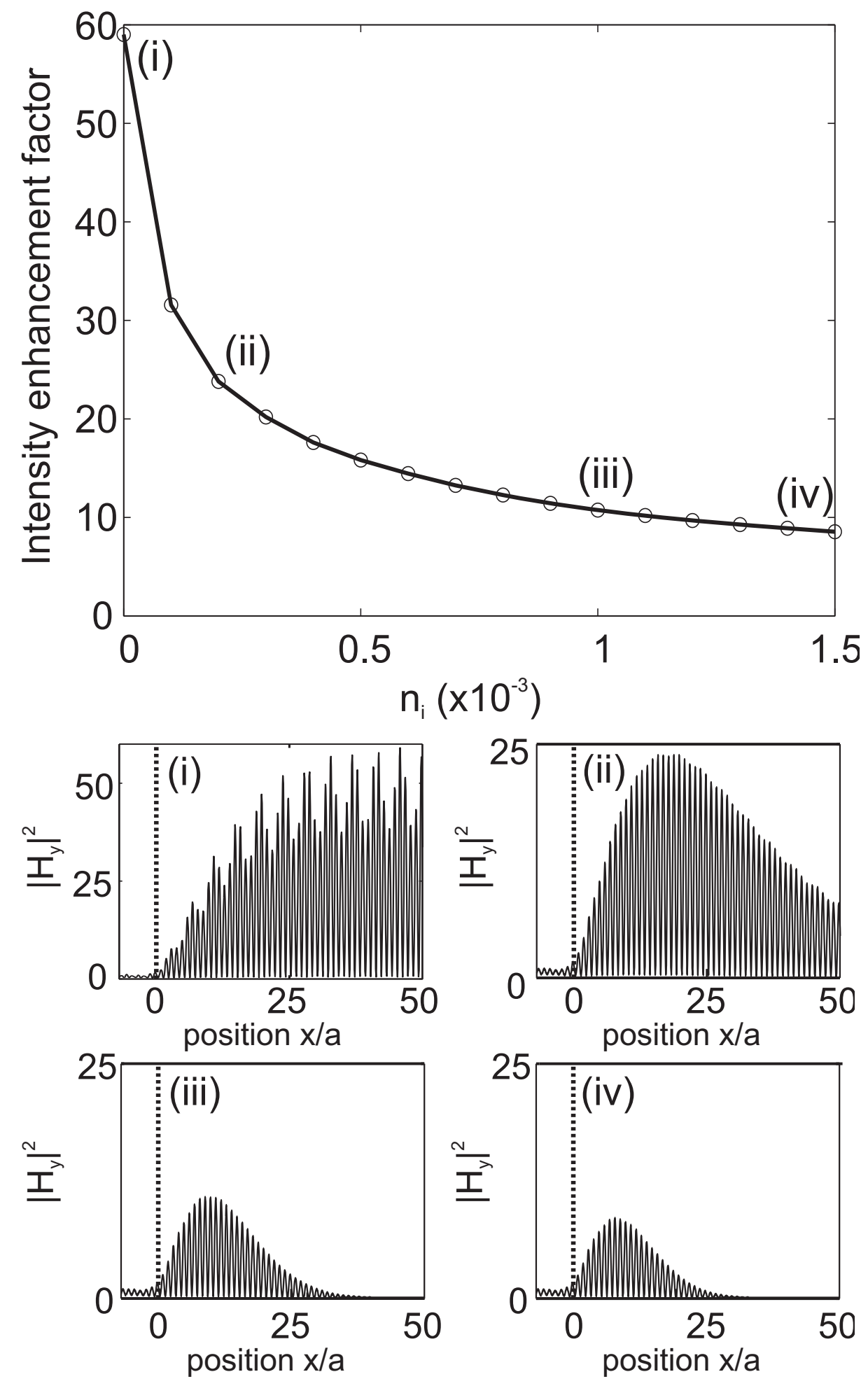

Figure 4. Top: field enhancement in the slow light $\mathrm{PhC}$ waveguide as a function of $n_{i}$. (i)-(iv): $\left|H_{y}\right|^{2}$ field on the waveguide axis at points (i)-(iv) marked on the top plot. 
5. T. P. White, L. C. Botten, C. M. de Sterke, K. B. Dossou, and R. C. McPhedran, "Efficient slow-light coupling in a photonic crystal waveguide without transition region," Opt. Lett. 33, pp. 2644-2646, Nov 15 2008.

6. J. Ballato, A. Ballato, A. Figotin, and I. Vitebskiy, "Frozen light in periodic stacks of anisotropic layers," Phys. Rev. E 71, pp. 036612-12, Mar 2005.

7. L. OFaolain, S. A. Schulz, D. M. Beggs, T. P.White, M. Spasenovic, L. Kuipers, F. Morichetti, A. Melloni, S. Mazoyer, J. P. Hugonin, P. Lalanne, and T. F. Krauss, "Loss engineered slow light waveguides," Opt. Express 8, pp. 27627-27638, 2010.

8. N. Le Thomas, V. Zabelin, R. Houdre, M. V. Kotlyar, and T. F. Krauss, "Influence of residual disorder on the anticrossing of bloch modes probed in k space," Phys. Rev. B 78, pp. 125301-8, Sep 2008.

9. J. Grgic, J. Pedersena, S. Xiaoa, and N. Mortensen, "Group index limitations in slow-light photonic crystals," Photonics Nanostruct.: Fundam. Appl. 8, pp. 56-61, 2010.

10. J. Grgic, S. Xiao, J. Mork, A.-P. Jauho, and N. A. Mortensen, "Slow-light enhanced absorption in a hollowcore fiber," Opt. Express 8, pp. 14270-14279, 2010.

11. J. P. Hugonin, P. Lalanne, T. P. White, and T. E. Krauss, "Coupling into slow-mode photonic crystal waveguides," Opt. Lett. 32, pp. 2638-2640, Sep 152007.

12. S. Ha, A. A. Sukhorukov, K. B. Dossou, L. C. Botten, C. M. de Sterke, and Y. S. Kivshar, "Bloch-mode extraction from near-field data in periodic waveguides," Opt. Lett. 34, pp. 3776-3778, Dec 152009.

13. B. Corcoran, C. Monat, C. Grillet, D. J. Moss, B. J. Eggleton, T. P. White, L. O'Faolain, and T. F. Krauss, "Green light emission in silicon through slow-light enhanced third-harmonic generation in photonic-crystal waveguides," Nature Photonics 3, pp. 206-210, Apr 2009. 